\title{
Hermes
}

Published online: 13 September 2021

(C) Springer-Verlag GmbH Germany, part of Springer Nature 2021

Fetal and perinatal outcome following first and second trimester COVID-19 infection: evidence from a prospective cohort study

Rosen H, Bart Y, Zlatkin R et al

J Clin Med (2021) 10:2152

The authors studied 55 pregnant women with proven severe acute respiratory syndrome coronavirus 2 (SARS-CoV-2) infection contracted prior to 26 weeks of gestation. The goal was to investigate the effect of first- and second-trimester maternal SARS-CoV-2 infection on fetal and perinatal outcomes. Serial prenatal US was performed every 4-6 weeks and fetal MRI at 30-32 weeks. All mothers were either asymptomatic or presented with mild symptoms. None of the fetuses had findings of central nervous system disease, growth restriction or placental dysfunction on imaging. Twenty-two of the mothers had amniocentesis performed, and none showed evidence of vertical transmission of the virus. Perinatal survival was $100 \%$. Two neonates with negative fetal SARS-CoV-2 sampling had major anomalies that were not considered to be linked to the SARS-CoV-2 infection (anhydramnios with small hyperechoic kidneys, and fetal cataract). The authors concluded that SARS-CoV-2 infection at early gestation in asymptomatic or mildly symptomatic mothers was not proved to be associated with vertical transmission and did not impact obstetric and neonatal outcomes.

The importance of heart and brain imaging in children and adolescents with multisystem inflammatory syndrome in children (MIS-C)

Mavrogeni SI, Kolovou G, Tsirimpis $V$ et al

Rheumatol Int (2021) 41:1037-1044

Multisystem inflammatory syndrome in children (MIS-C) is progressively recognized as a serious complication of severe acute respiratory syndrome coronavirus 2 (SARS-CoV-2) infection. The clinical presentation can mimic Kawasaki disease. A relatively high percentage of children with MIS-C and Kawasaki-like symptoms require intensive care support.
Fever, mucocutaneous findings, myocardial dysfunction (in particular left ventricular), cardiac conduction abnormalities, shock, gastrointestinal symptoms, respiratory signs and lymphadenopathy can be seen in MIS-C. Cardiac MRI might show diffuse myocarditis with T2-hyperintense edema, hyperemia and early capillary leakage of contrast agent as well as signs of fibrosis on delayed imaging. Furthermore, mild coronary artery dilatation and ectasia have been reported as complications of MIS-C. Consequently, cardiac MRI or CT angiography should be considered in these children.

Features of intestinal disease associated with COVIDrelated multisystem inflammatory syndrome in children Sahn B, Eze OP, Edelman MC et al JPGN (2021) 72:384-387

The authors of this study investigated the clinical manifestations of gastrointestinal disease in children affected with multisystem inflammatory syndrome in children (MIS-C). Thirtyfive children who presented with abdominal pain and/or diarrhea and met the diagnostic criteria of the Centers for Disease Control (CDC) for MIS-C were evaluated for the study. The children received abdominal radiographs, and abdominal US or CT examinations. The radiographic features included bowel wall thickening, mesenteric fat stranding, and right lower quadrant mesenteric lymphadenopathy. In particular the distal ileum appeared to be affected, mimicking terminal ileitis/ inflammatory bowel disease (IBD). The authors found that more than $95 \%$ of the children in their cohort presented with gastrointestinal symptoms and more than $50 \%$ of those had CT imaging findings consistent with terminal ileitis and bowel wall thickening. Luminal narrowing can result in partial or complete bowel obstruction requiring surgical resection. The histological features in MIS-C appeared different from those found in children with chronic IBD as venous microthrombi and accompanying necrotizing lymphadenitis were noted in this cohort of patients. The authors concluded that the gastrointestinal tract of children with MIS-C appears especially prone to inflammatory damage. 
Checklist for responsible deep learning modeling of medical images based upon COVID-19 detection studies

Hryniewska W, Bombiński P, Szatkowski P et al

Pattern Recognit (2021) 118:108035

Artificial intelligence, machine learning and deep learning are promising developments in which a deep learning approach uses complex neural network models to extract important information from multiple databases that supersedes the human skills at the picture archiving and communication system (PACS) workstation. The authors analyzed the various models published in the recent literature using lung image data for detecting coronavirus disease 2019 (COVID-19). Their analysis critically evaluated the various errors that might impact the modeling. These include scarcity of publicly available COVID-19 data sources with images in raw Digital Imaging and Communications in Medicine (DICOM) format, too few images with low and moderate severity cases, relatively low number of COVID-19 images, data sources lacking descriptions, mixture of CT and radiographic images, inappropriate CT windows, incorrect categorization of pathologies, lack of information about chest projection for radiographic imaging, and mixture of pediatric and adult imaging data sets. The authors correctly pointed out that there are crucial differences between the chest radiographs of children and those of adults because of technical, anatomical and pathological differences. This review emphasized the importance of well-defined and reliable data input and thorough model validation. In addition, the authors found that radiologists should be consulted and involved in modeling projects.

\section{The era of virtual care: perspectives of youth on virtual appointments in COVID-19 and beyond}

Fletcher SE, Tsang VWL

Paediatr Child Health (2021) 26:210-213

The coronavirus disease 2019 (COVID-19) pandemic has generated a surge in interest in the practice of telemedicine. The authors of this manuscript, operating under the Maternal, Infant, Child and Youth Research Network in Canada, studied the value, benefits and drawbacks of virtual care by querying
10 individuals 13-17 years of age who were representative of health care experiences and geographic and demographic characteristics. The authors collected relevant data and discussion points during two semi-structured discussions in June and October 2020. Virtual care visits were progressively rolled out because of COVID-19-related social distancing and the need to reduce travel to limit virus spread. Virtual visits were facilitated by many technological advancements and the progressive technical literacy of young patients. This study identified several benefits of virtual care as reported by these young individuals. These included ease of accessibility, time saved by limiting travel to the hospital, ease of coordination with school schedule, ease of incorporation of working parents into the hospital visits, elimination of clinic waiting time, and receiving care from the comfort of their home. Furthermore, the young people thought that virtual care could facilitate continuity of care, mitigate health-related stress and decrease transmission risks. On the flip side, privacy limitations were reported as a major concern because most youth did not have space within their home to participate in virtual appointments without parents or other family members overhearing or interrupting. They also reported hesitation to accurately describe symptoms and signs to the same degree as in an inperson interaction. The youths were also concerned about certain aspects of care that can only be provided with an inperson hospital or practice visit, such as laboratory workup or imaging. The young people mentioned that they would feel more comfortable with virtual visits after having established a relationship with their physician. Finally, the authors noted that the possibility of limited technological or internet accessibility to young patients or families should not be ignored.

Abstracted by: Thierry A. G. M. Huisman E-mail: huisman@texaschildrens.org

Publisher's note Springer Nature remains neutral with regard to jurisdictional claims in published maps and institutional affiliations. 